CASE REPORT

\author{
R. Arnold \\ C. Johnson \\ B. McNulty
}

G. Gaisie

\title{
Substantia Nigra MR Imaging Signal Changes and Cardiomyopathy Following Prenatal Exposure to Cocaine and Heroin
}

SUMMARY: Exposure to cocaine in utero results in behavioral and neurodevelopmental abnormalities that persist into adulthood. Conventional MR imaging has generally failed to reveal the expected structural lesions to explain these clinical findings. We report a case of focal MR imaging signalintensity changes in the substantia nigra, locus ceruleus, and other selected nerve tracts and nuclei in a child exposed prenatally to cocaine and other drugs. The patient also had dilated cardiomyopathy.

M aternal cocaine use during pregnancy is a widespread problem, which leads to a spectrum of behavioral, cognitive, and motor dysfunctions. Animal and human studies have attempted to define the specific neurologic centers damaged by prenatal cocaine exposure. Since 1991, attempts to document MR imaging changes in the brains of animals and humans exposed to cocaine in utero have produced mixed results. Findings of brain MR imaging of exposed children are generally reported to be anatomically normal. ${ }^{1,2}$

The substantia nigra, locus ceruleus, ventral caudate, and ventral tegmental area have been implicated as focal targets of cocaine, resulting in neuronal activation, reduced cell attenuation, and apoptosis. ${ }^{3,4}$ Additionally, prenatal exposure to cocaine has been associated with cardiac arrhythmias, congestive heart failure, cardiorespiratory arrest, and death. ${ }^{5}$ We present a case of MR imaging changes in the substantia nigra and several other focal midbrain and brain stem centers in a child exposed to cocaine and heroin in utero. The patient also had severe left ventricular dysfunction at presentation.

\section{Case Report}

The mother of our patient used crack cocaine, heroin, alcohol, and prescription quetiapine fumarate and smoked 2 packs of cigarettes per day throughout pregnancy. Methamphetamine abuse was also mentioned in 1 emergency department report. The patient was born full term via cesarean delivery, weighing 7 pounds, 13 ounces $(3.5 \mathrm{~kg}$ ). Apgar scores were 9 and 9 at 1 and 5 minutes. The patient was in the care of his biologic family for the first 2-3 months of life, but physical abuse and neglect were suspected during this time. He was removed from the home and was eventually placed in foster care.

The patient was referred to our center for failure to thrive with longstanding developmental and growth delays. At the time of presentation to pediatric neurology, the patient was 15 months old. On the basis of the Provence Birth to Three Developmental Profile, gross motor skills were at a 5-month level; language skills were at a 9-month

Received November 5, 2007; accepted after revision December 5.

From the Departments of Diagnostic Radiology (R.A., C.J.) and Neuroradiology (B.N.), Northeastern Ohio Universities College of Medicine-Canton Affiliated Hospitals, Canton, Ohio; and the Department of Pediatric Radiology (G.G.), Akron Children's Hospital, Akron, Ohio.

This manuscript has received a waiver from the hospital IRB committee and consent was obtained from the parents.

Please address correspondence to Ryan Arnold, MD, Department of Diagnostic Radiology, Northeastern Ohio Universities College of Medicine-Canton Affiliated Hospitals, 2600 Sixth St SW, Canton, OH 44710; e-mail: ryanradiology@gmail.com

DOI 10.3174/ajnr.A0966 level; and fine motor, cognitive, self-help, and social/emotional skills were at a 10 -month level.

Neurologic examination demonstrated mildly reduced muscle tone, a persistent truncal incurvation reflex, lack of lateral and anterior propping reflex, and dynamic spasticity of the hands, with the tendency to keep them closed. Deep tendon reflexes were symmetric, and Babinski reflexes were absent. Fine and gross motor tremors were variably reported by different observers, not including the patient's pediatric neurologist. Ophthalmologic evaluation revealed an oculomotor apraxia, evidenced by the use of head movements rather than eye motion to track objects visually.

MR imaging of the brain performed without contrast revealed elevated T2 signal intensity within the pars compacta of the substantia nigra (Fig 1A), with corresponding low T1 signal intensity and heterogeneous intermediate-to-high fluid-attenuated inversion recovery signal intensity. Focal areas of similar abnormal signal intensity were seen in the dorsal pons, the dorsal medulla, and the upper cervical spinal cord. These abnormalities encompassed the motor nucleus of the vagus nerve (Fig $1 B$ ), solitary tract and nucleus (Fig $1 C$ ), medial vestibular nucleus (Fig $1 D$ ), and the posterior horns and central portions of the upper cervical spinal cord (not shown). Patchy areas of abnormal T2 signal intensity were also seen in the thalamus. There was mildly increased T2 signal intensity in the region of the locus ceruleus. Diffusion-weighted imaging was normal.

An echocardiogram revealed a dilated left ventricle with a left ventricular ejection fraction of $17 \%$. The patient was transferred to a cardiac transplantation center for further work-up.

\section{Discussion}

Many studies have attempted to localize and characterize damage caused by in utero exposure to cocaine and other drugs of abuse. Conventional MR imaging techniques have generally failed to reveal structural abnormalities in these patients; indeed, most series report normal findings on MR imaging examinations. One case of diffuse severe pachygyria following prenatal cocaine exposure has been reported. ${ }^{6}$ Advanced neuroimaging techniques have recently been used with some success. MR spectroscopy has shown increased creatine levels in the frontal white matter, with trends for decreased midbrain volumes. ${ }^{2}$ Increased diffusion was shown with diffusion tensor imaging in the left frontal callosal and right frontal projection fibers of cocaine-exposed children. ${ }^{7}$

The developing dopaminergic system is a substrate for cocaine in utero, and trends toward decreased cell densities in the substantia nigra have been reported in prenatal cocaine exposure in primates. ${ }^{4}$ Prolonged cocaine exposure through- 

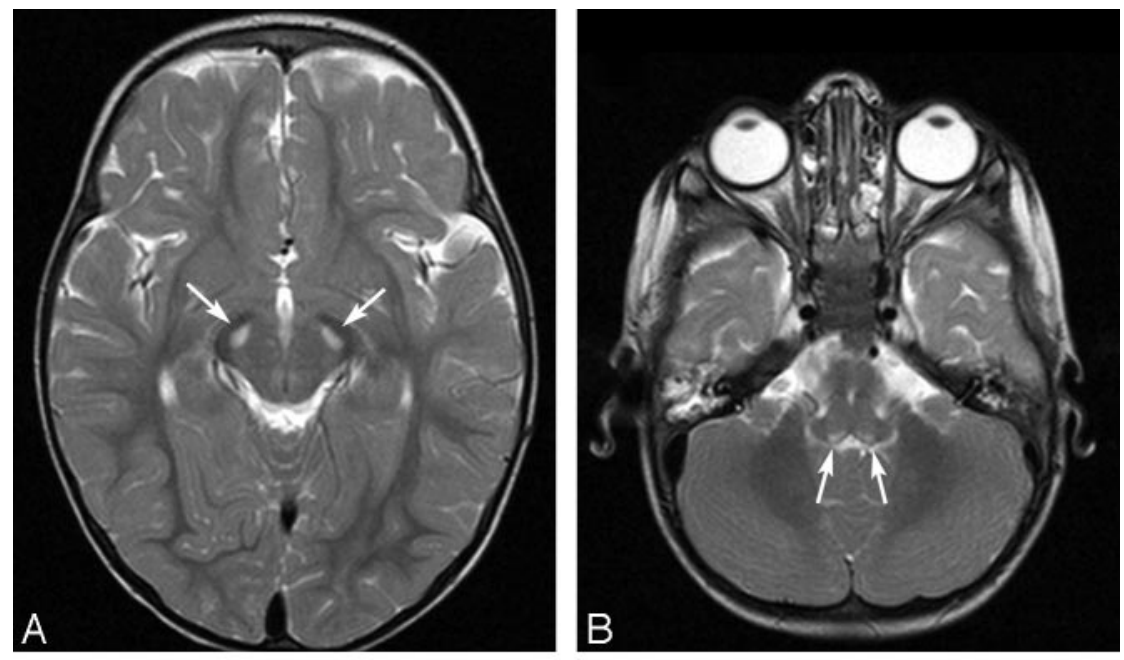

Fig 1. A T2-weighted axial sequence demonstrates symmetric well-circumscribed hyperintensities (arrows) within the pars compacta of the substantia nigra $(A)$. T2-weighted images of the dorsal motor nucleus of the vagus $(B)$, the solitary nucleus $(C)$, and the medial vestibular nucleus $(D)$ demonstrate similar symmetric well-circumscribed focal hyperintensities (arrows).

more severe. The caudate, putamen, and globus pallidus are typically affected in Leigh disease. These areas were spared in our patient.

The role of prenatal exposure to methamphetamine, alcohol, and quetiapine was also individually contemplated. Although our patient shared several characteristics of each of these entities, none of them can independently explain the clin-
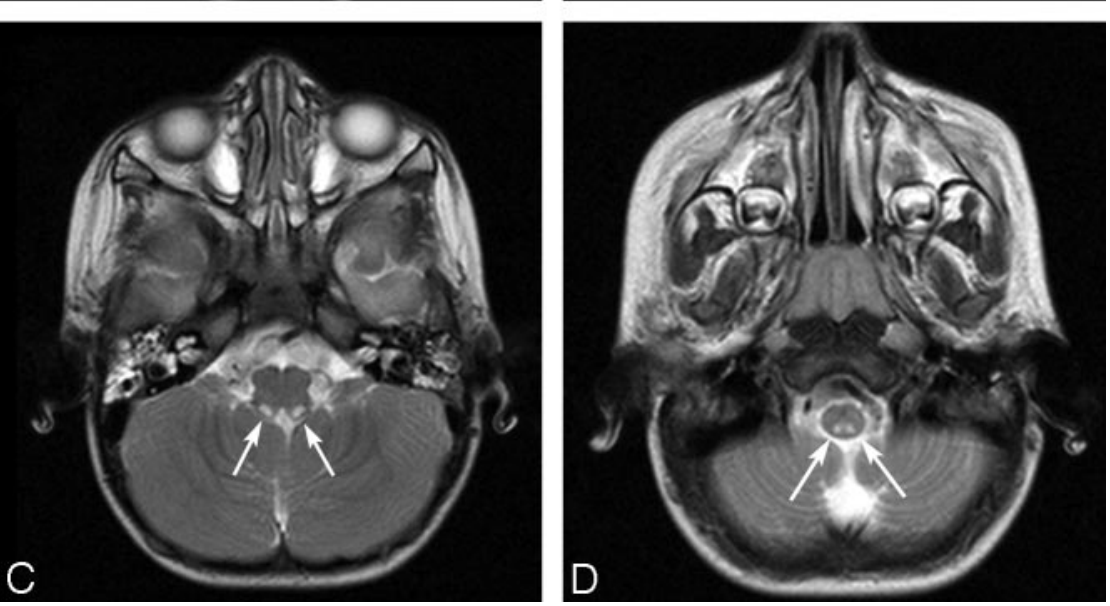
ical and radiologic findings described in this patient. However, several authors have proposed interactive or potentiating effects of various drugs as an important precursor in producing toxic changes severe enough to be seen with standard imaging. ${ }^{1,7}$ Quetiapine, a dopamine antagonist, deserves special consideration as a possible potentiator in this case.

In summary, signal-intensity abnormalities in the substantia nigra following cocaine exposure are not surprising on the basis of animal models, yet these changes have not been described in MR imaging studies in humans. A combination of cocaine, heroin, quetiapine, alco-

out development of this area is a compelling explanation for the signal intensity abnormalities we describe. Cocaine is concentrated in fetal blood, with fetal/maternal plasma ratios reported as high as 5:1 in primates. ${ }^{4}$ Cocaine also induces apoptosis in the locus ceruleus of rats in utero. ${ }^{3} \mathrm{MR}$ imaging changes to this region were seen in our patient.

Inhalation of heroin fumes, known as "chasing the dragon," has resulted in spongiform leukoencephalopathy, with focal MR imaging signal intensity characteristics similar to those seen in our patient. Signal intensity abnormalities of the solitary tract, as seen in our patient, have been documented in these heroin abusers. ${ }^{8}$ Unlike other reports of heroin-induced leukoencephalopathy, however, there was sparing of the internal capsule and cerebellar white matter in our patient.

Alternative explanations for the MR imaging findings that were considered included global hypoxic events and neurometabolic disorders such as Leigh disease (subacute necrotizing encephalomyelopathy). With global ischemia, focal damage to the substantia nigra and thalamus can occur, but injury to the cortex, hippocampus, caudate, and putamen is generally hol, and tobacco in this patient probably played a role in the abnormalities we report.

\section{References}

1. Link EA, Weese-Mayer DW, Byrd SE. Magnetic resonance imaging in infants exposed to cocaine prenatally: a preliminary report. Clin Pediat (Phila) 1991;30:506-08

2. Smith L, Chang L, Yonekura M, et al. Brain proton magnetic resonance spectroscopy and imaging in children exposed to cocaine in utero. Pediatrics 2001;107:227-31

3. Dey $\mathrm{S}$, Mactutus $\mathrm{C}$, Booze $\mathrm{R}$, et al. Cocaine exposure in vitro induces apoptosis in fetal locus coeruleus neurons by altering the $\mathrm{Bax} / \mathrm{Bcl}-2$ ratio and through caspase-3 apoptotic signaling. Neuroscience 2007;144:509-21. Epub 2006 Nov 3

4. Lidow MS. Consequences of prenatal cocaine exposure in nonhuman primates. Brain Res Dev Brain Res 2003;147:23-36

5. Frassica J, Orav E, Walsh E. Arrhythmias in children prenatally exposed to cocaine. Arch Pediatr Adolesc Med 1994;148:1163-69

6. Gomez-Anson B, Ramsey R. Pachygyria in a neonate with prenatal cocaine exposure: MR features. J Comput Assist Tomogr 1994;18:637-39

7. Warner T, Behnke M, Eyler F. Diffusion tensor imaging of frontal white matter and executive functioning in cocaine-exposed children. Pediatrics 2006; 118:2014-24

8. Hagel J, Andrews G, Vertinsky T, et al. Chasing the dragon: imaging of heroin inhalation leukoencephalopathy. Can Assoc Radiol J 2005;56:199-203 